Bull. Mater. Sci., Vol. 23, No. 4, August 2000, pp. 273-279. (C) Indian Academy of Sciences.

\title{
Conductivity, dielectric behaviour and magnetoelectric effect in copper ferrite-barium titanate composites
}

\author{
R P MAHAJAN, K K PATANKAR, M B KOThale and S A PATIL* \\ Department of Physics, Shivaji University, Kolhapur 416 004, India
}

MS received 27 December 1999; revised 22 May 2000

\begin{abstract}
Composites of $\mathrm{CuFe}_{2} \mathrm{O}_{4}$ and $\mathrm{BaTiO}_{3}$ were prepared using a conventional ceramic double sintering process. The presence of both phases was confirmed by $X$-ray diffraction. The variations of resistivity and thermo emf with temperature in these samples were studied. All the composites showed $n$-type behaviour. The variation of dielectric constant $\left(\varepsilon^{\prime}\right)$ in the frequency range $100 \mathrm{~Hz}$ to $1 \mathrm{MHz}$ and with temperature at constant frequency were studied. The conduction phenomenon was explained on the basis of a small polaronhopping model. Also confirmation of this phenomenon was made with the help of a.c. conductivity measurements. The static value of the magnetoelectric conversion factor, i.e. d.c. $(\mathrm{ME})_{\mathrm{H}}$ was studied as a function of intensity of the magnetic field. The maximum value of ME coefficient was observed for $75 \%$ ferroelectric phase composite.
\end{abstract}

Keywords. Conductivity; dielectric behaviour; magnetoelectric effect; $\mathrm{CuFe}_{2} \mathrm{O}_{4} ; \mathrm{BaTiO}_{3}$.

\section{Introduction}

Magnetoelectric composites consist of two phases viz. piezoelectric and piezomagnetic. The ME effect is a coupled two-field effect in which application of an electric field induces magnetization and a magnetic field induces electrical polarization. The ME effect is a property of the composites, which is absent in their constituent phases (Ce-Wen 1994). In his paper Van Suchtelen (1972) mentioned a magnetoelectric (ME) composite material being obtained by unidirectional solidification of a $\mathrm{BaTiO}_{3}-\mathrm{CoFe}_{2} \mathrm{O}_{4}$ eutectic liquid. The deformation of ferrite phase causes the polarization of piezoelectric particles of the composite material. However the electrical polarization of the piezoelectric material causes a change in magnetization of ferrite phase due to mechanical coupling of piezomagnetic and piezoelectric phases (Lopatin 1994). ME conversion in ferrite-barium titanate composites was experimentally reported by Boomgaard et al (1978).

The composites can be prepared either by unidirectional cooling or by solid state reaction. Moreover sintered composites are much cheaper and easier to prepare than the unidirectionally cooled ones. Literature survey indicates that not much detailed work on the physical properties has been done in these magneto ferroelectric composites. The work available in literature is confined to the measurement of ME effect only. Schmid (1994) has mentioned important applications of ME effect. Accord-

*Author for correspondence ing to his analysis ME coefficient of composites largely depends on equilibrium of two phases: perfect mechanical coupling between grains and resistivity of composites. However, until now no conclusive theory has been formulated for the conduction mechanism in these materials. Parameters such as thermoelectric power and electrical resistivity are the most convenient to measure and suggest the conduction phenomenon and identify the charge carriers responsible for conduction. Hence it is necessary to study in detail the electrical behaviour and dielectric studies of these composites.

Unusual thermal magnetic and dielectric properties of copper ferrite and mixed copper ferrite have been reported (Nanba and Kobayashi 1978; Patil et al 1996). In general these properties have been attributed to Jahn Teller distortion of $\mathrm{Cu}$ ions in the ferrites. Therefore it is hoped that the Jahn Teller distortion of $\mathrm{Cu}$ ferrite may induce mechanical coupling in the composite, which is a requirement for $\mathrm{ME}$ effect.

In this paper we report the results on electrical resistivity, thermo electric power, dielectric behaviour and $\mathrm{ME}$ coefficient of $\mathrm{CuFe}_{2} \mathrm{O}_{4}+\mathrm{BaTiO}_{3}$ composites to understand conduction mechanism and dependence of $(\mathrm{d} E / \mathrm{d} H)_{\mathrm{H}}$ on composition and magnetic field.

\section{Experimental}

The samples were characterized by using X-ray diffractometer (XRD) (Phillips model 1710/pw1710) using $\mathrm{CuK} \alpha$ radiation (Ni filter). Microstructural studies of fractured discs were carried out using scanning electron micro- 
scope. The electrical measurements were performed by means of a two-probe method. A low value of constant voltage was applied across the sample and the current through the sample was measured as a function of temperature in the range $300-800 \mathrm{~K}$. The Seebeck coefficient $(\alpha)$ is given by the relation

$$
\alpha=\Delta V / \Delta T
$$

where $\Delta V$ is voltage measured across the sample, $\Delta T$ the temperature difference across the pellet. A temperature difference $\Delta T \cong 20^{\circ} \mathrm{C}$ was maintained across the pellet with microfurnace fitted to the sample holder assembly. Temperatures of both the surfaces of the pellet were measured with Chromel-Alumel thermocouple. Seebeck coefficient was measured as a function of temperature in the range $300-800 \mathrm{~K}$.

The dielectric measurements were carried out in the range from $100 \mathrm{~Hz}$ to $1 \mathrm{MHz}$ and with temperature at fixed frequency $10 \mathrm{KHz}$ by using LCR meter bridge (HP LCR 4284A). The dielectric constant ( $\left.\varepsilon^{\prime}\right)$ was calculated by using the formula

$$
\varepsilon^{\prime}=C^{*} d / \varepsilon_{0} A
$$

where $C$ is capacitance of pellet in farad, $d$ the thickness of pellet in meter, A the cross-sectional area of the flat surface of the pellet and $\varepsilon_{0}$ a constant of permittivity of free space. To obtain ME signal the samples have to be poled electrically. For electrical poling the samples were heated above their ferroelectric transition temperature i.e. $>150^{\circ} \mathrm{C}$, with applied high electric field of about $3.5 \mathrm{kV}$ and then allowed to cool slowly in presence of electric field. Magnetic poling was carried out by keeping the sample in d.c. magnetic field for $15 \mathrm{~min}$ in the saturation field value of $1.5 \mathrm{KOe}$. For measurement of ME output a specially designed perspex sample holder with copper electrodes was used (Suryanarayana 1994) and voltage developed across the samples was measured with varying the d.c. magnetic field.

\subsection{Preparation of ME composites}

ME composite contains ferrimagnetic and ferroelectric phases. The ferrite phase chosen was copper ferrite, and ferroelectric phase was barium titanate. Copper ferrite was prepared by standard ceramic technique starting with AR grade $\mathrm{CuO}$ and $\mathrm{Fe}_{2} \mathrm{O}_{3}$ in molar ratios. Similarly the ferroelectric phase i.e. $\mathrm{BaTiO}_{3}$, was prepared from $\mathrm{BaO}$ and $\mathrm{TiO}_{2}$. These raw materials were mixed thoroughly. The constituent phases were presintered at $750^{\circ} \mathrm{C}$ for $6 \mathrm{~h}$. After presintering, the raw material was ground to fine powder. The composites were prepared by taking $\mathrm{CuFe}_{2} \mathrm{O}_{4}$ and $\mathrm{BaTiO}_{3}$ (ferroelectric concentration molar $25 \%, 50 \%$, $75 \%$ ). These compositions were mixed thoroughly and presintered at $900^{\circ} \mathrm{C}$ for $6 \mathrm{~h}$. Then the presintered powder was pressed into pellets of dimension $10 \mathrm{~mm}$ diameter

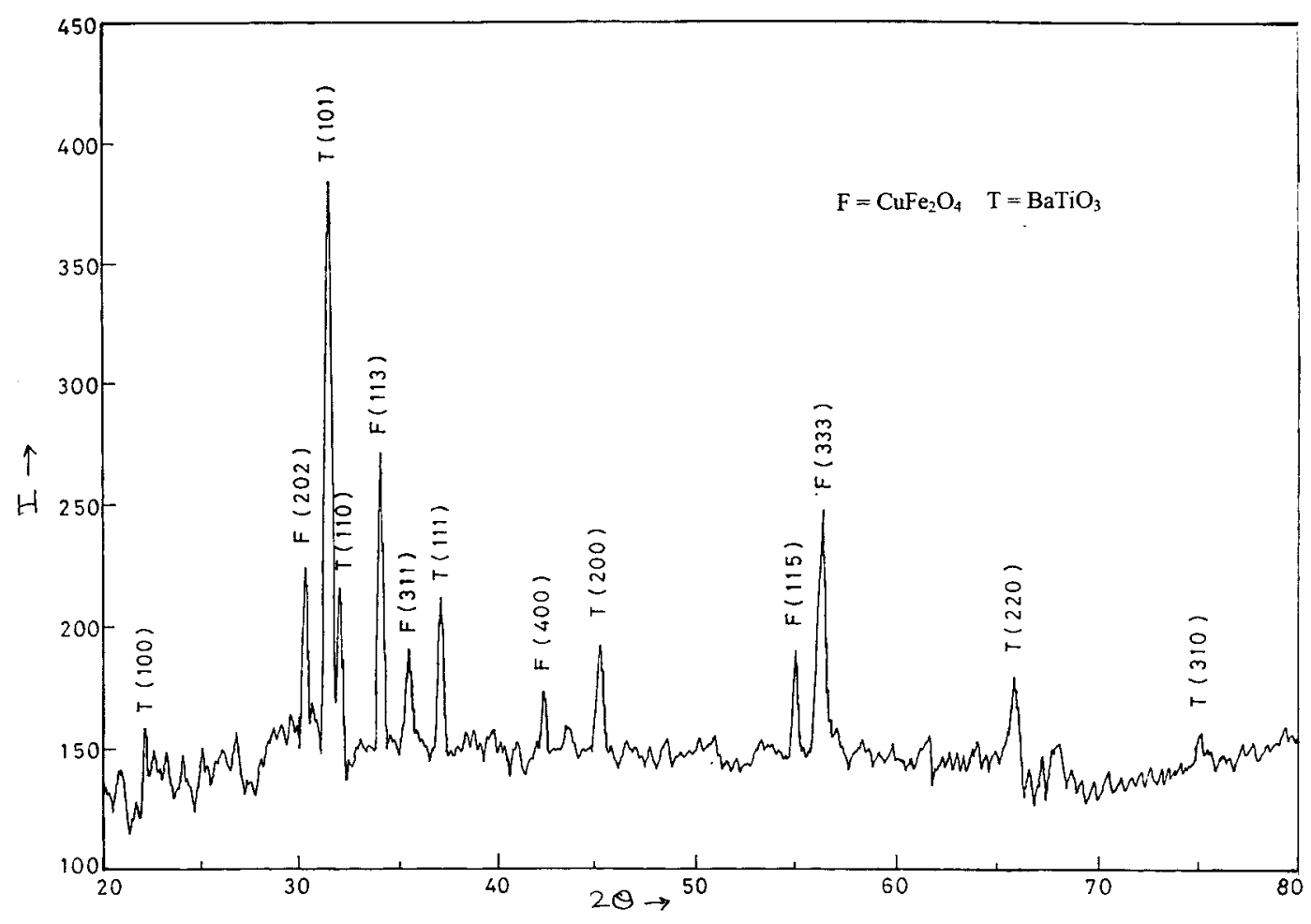

Figure 1. X-ray diffractogram of composite containing 50\% ferroelectric and 50\% ferromagnetic phase. 

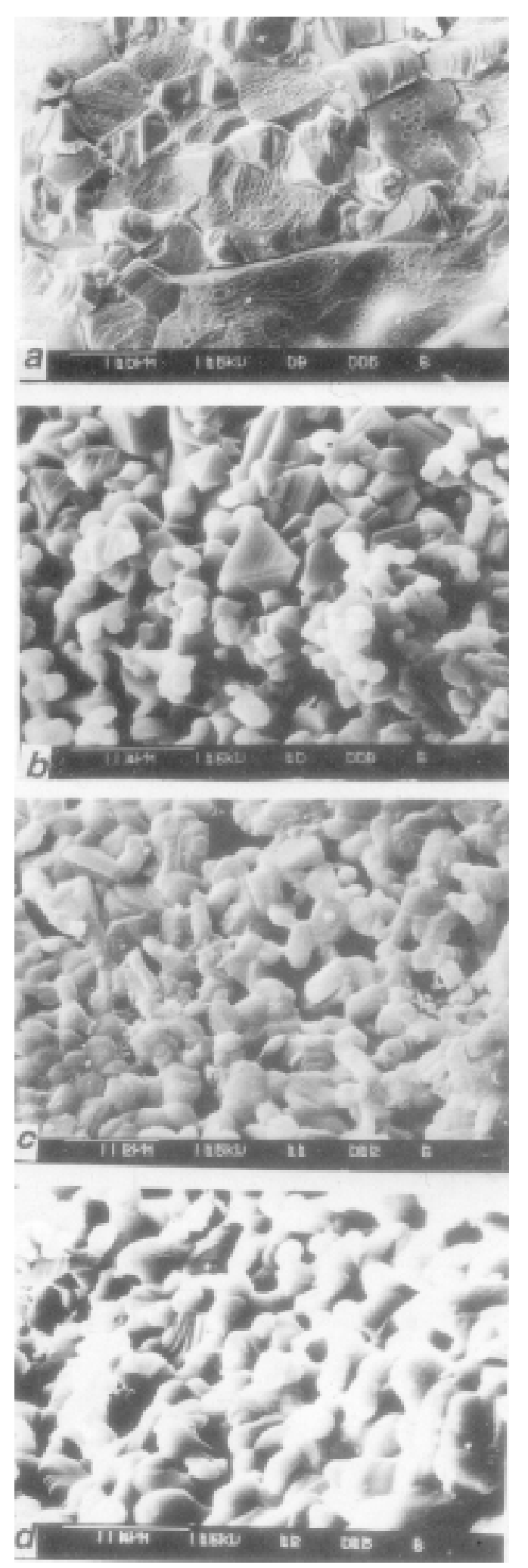

Figure 2. $\mathrm{SEM}$ for the $\mathrm{CuFe}_{2} \mathrm{O}_{4}+\mathrm{BaTiO}_{3}$ composites: a. $\mathrm{CaFe}_{2} \mathrm{O}_{4}$; b. $75 \% \mathrm{CuFe}_{2} \mathrm{O}_{4}+25 \% \mathrm{BaTiO}_{3}$, c. $50 \% \mathrm{CuFe}_{2} \mathrm{O}_{4}+$ $50 \% \mathrm{BaTiO}_{3}$ and d. $25 \% \mathrm{CuFe}_{2} \mathrm{O}_{4}+75 \% \mathrm{BaTiO}_{3}$. and $3 \sim 4 \mathrm{~mm}$ thickness. The pelletized samples were finally sintered at $1200^{\circ} \mathrm{C}$ for $12 \mathrm{~h}$ and slowly cooled to room temperature at the rate of $80^{\circ} \mathrm{C} / \mathrm{h}$.

\section{Results and discussion}

The diffraction pattern shown in figure 1 reveals that the composites consist of copper ferrite and barium titanate as predominant phases. The intensity of barium titanate peak (110 plane) increases with increasing percentage of barium titanate. The lattice constants of individual phases do not vary much. The tetragonal structure of copper ferrite is retained in all composites. The values of lattice constants for present composites are given in table 1. Figure 2 shows SEM micrographs of present system. The values of average grain diameter are given in table 1 . It is noted that the average grain diameter decreases with increasing $\mathrm{BaTiO}_{3}$ concentration. The composites of 50 and $75 \%$ exhibit rod type structure.

The variation of DC resistivity for the composites with temperature in the range $300-800 \mathrm{~K}$ is shown in figure 3 . The relationship between resistivity and temperature may be expressed as (Verwey and Heilman 1947),

$$
\rho=\rho_{0} \exp (\Delta E / K T)
$$

where $\rho$ is resistivity at temperature $T, \Delta E$ the activation energy for electrical process.

From these plots it is observed that all the samples show three regions with changing slope at different temperatures. It is suggested that the conduction mechanism changes from one region to another region. The variation of resistivity was explained on the basis of actual location of cation in the spinel structure. Conduction in ferrite is due to electron transfer between divalent and trivalent ions. The conduction at low temperature (i.e $<450 \mathrm{~K}$ ) is due to impurities, whereas at higher temperature (i.e. $>400 \mathrm{~K})$ due to polaron hopping. The calculated activation energies $(\Delta E)$ are greater than $0.4 \mathrm{eV}$ which clearly suggest that the conduction is due to polaron hopping (Klinger 1975). The result of conduction by hopping process is large effective mass and low mobility to current carriers. The temperature dependence of electrical conductivity in such a case involves less of temperature dependent concentration of carriers and mostly associated with temperature dependent mobility. The activation energies calculated from resistivity are noted in table 1.

Figure 4 shows the variation of $\alpha$ with temperature for $\mathrm{CuFe}_{2} \mathrm{O}_{4}$ composites. It is observed that the variation of Seebeck coefficient $(\alpha)$ with temperature for $\mathrm{CuFe}_{2} \mathrm{O}_{4}$ shows $p$ type conduction up to $450 \mathrm{~K}$ and $p-n$ transition takes place above $450 \mathrm{~K}$. This may be due to structural changes which occurred in the $\mathrm{CuFe}_{2} \mathrm{O}_{4}$ (Patil 1980). It has been already reported in the literature that copper ferrite exhibits $p$-type conduction (Kumar Krishan et al 
1987) attributed to appearance of $p$ carriers due to reduction of $\mathrm{Cu}^{2+}$ ions to $\mathrm{Cu}^{1+}$ at about $900-950^{\circ} \mathrm{C}$ during sintering process (Stierstadt et al 1964). All the composites show $n$-type conduction throughout the temperature range. A cusp is observed at about $550^{\circ} \mathrm{K}$, which is due to oxidation-reduction process. At lower temperature ( $<400 \mathrm{~K})$ the $\alpha$ value increases with temperature. The majority of charge carriers are electrons generated from $\mathrm{Fe}^{2+}$ ions, which act as donor centres. The temperature dependence of $\alpha$ may be attributed to predominance of impurity conduction in this region $(<400 \mathrm{~K})$, while it is due to polaron hopping at higher temperature $(>400 \mathrm{~K})$. There is a strong experimental proof for the existence of polarons and their hopping process (Klinger 1975). The activation energies calculated from conductivity data are much larger than the values required for $\mathrm{Fe}^{3+} \Leftrightarrow \mathrm{Fe}^{2+}$ tran- sition in ferrites. In the ferrites the conduction phenomena is explained on the basis of small polaron hopping model.

The drift mobility $\mu_{\mathrm{d}}$ was calculated in the higher temperature region i.e. $>450 \mathrm{~K}$, using the data of $\rho$ and $\alpha$ and applying the method proposed by Eatch et al (1987) at different temperatures for all compositions. The relation for drift mobility is given by

$$
\exp (\alpha /(2 \cdot 3 K / e)) / \rho=2 N_{0} e \mu_{\mathrm{d}}
$$

The notations have their usual meaning.

We have calculated the mobility values from the above relation. The plots of $\log \mu_{\mathrm{d}}$ with reciprocal of temperature are shown in figure 5 . The activation energies are calculated and noted in table 1. It is observed that $\Delta E$ values closely agree with conductivity data. This is also

Table 1. Electrical data for the $\mathrm{CuFe}_{2} \mathrm{O}_{4}-\mathrm{BaTiO}_{3}$.

\begin{tabular}{|c|c|c|c|c|c|c|c|c|c|c|}
\hline \multirow[b]{2}{*}{ No. } & \multirow[b]{2}{*}{ Composition } & \multicolumn{2}{|c|}{ Lattice parameter $(\AA)$} & \multirow{2}{*}{$\begin{array}{l}\text { Grain dia. } \\
\qquad(\mu \mathrm{m})\end{array}$} & \multirow{2}{*}{$\begin{array}{c}\rho_{100^{\circ} \mathrm{C}} \\
(\mathrm{ohm}-\mathrm{m})\end{array}$} & \multirow[b]{2}{*}{$\alpha \mu \mathrm{V} / \mathrm{K}$} & \multicolumn{2}{|c|}{$\Delta E(\mathrm{eV})$} & \multirow{2}{*}{$\begin{array}{c}\mathcal{E}^{\prime} \text { at } \\
1 \mathrm{kHz}\end{array}$} & \multirow{2}{*}{$\begin{array}{c}(\mathrm{d} E / \mathrm{d} H)_{\mathrm{H}} \\
(\mu \mathrm{V} / \mathrm{cm} . \mathrm{Oe})\end{array}$} \\
\hline & & Ferrite & Ferroelect. & & & & Resistivity & Mobility & & \\
\hline S1 & $\mathrm{CuFe}_{2} \mathrm{O}_{4}$ & $\begin{array}{l}a=8 \cdot 307 \\
c=8 \cdot 490\end{array}$ & - & $4 \cdot 00$ & $7 \cdot 5 \times 10^{5}$ & $24 \cdot 9$ & $0 \cdot 84$ & $0 \cdot 80$ & $31 \cdot 00$ & - \\
\hline $\mathrm{S} 2$ & $\begin{array}{l}75 \% \mathrm{CuFe}_{2} \mathrm{O}_{4} \\
+25 \% \mathrm{BaTiO}_{3}\end{array}$ & $\begin{array}{l}a=8 \cdot 268 \\
c=8 \cdot 840\end{array}$ & $\begin{array}{l}a=3 \cdot 399 \\
c=4 \cdot 037\end{array}$ & $1 \cdot 50$ & $6.4 \times 10^{5}$ & -87 & $0 \cdot 72$ & $0 \cdot 68$ & $43 \cdot 28$ & 200 \\
\hline S3 & $\begin{array}{l}50 \% \mathrm{CuFe}_{2} \mathrm{O}_{4} \\
+50 \% \mathrm{BaTiO}_{3}\end{array}$ & $\begin{array}{l}a=8 \cdot 272 \\
c=8 \cdot 890\end{array}$ & $\begin{array}{l}a=4 \cdot 001 \\
c=4 \cdot 040\end{array}$ & $1 \cdot 33$ & $2.9 \times 10^{5}$ & -63 & $0 \cdot 49$ & $0 \cdot 55$ & $126 \cdot 88$ & 340 \\
\hline S4 & $\begin{array}{l}25 \% \mathrm{CuFe}_{2} \mathrm{O}_{4} \\
+75 \% \mathrm{BaTiO}_{3}\end{array}$ & $\begin{array}{l}a=8 \cdot 272 \\
c=8 \cdot 800\end{array}$ & $\begin{array}{l}a=3.998 \\
c=4.036\end{array}$ & $0 \cdot 86$ & $1.2 \times 10^{6}$ & -52 & $0 \cdot 46$ & $0 \cdot 46$ & $86 \cdot 58$ & 520 \\
\hline S5 & $\mathrm{BaTiO}_{3}$ & - & $\begin{array}{l}a=3.998 \\
c=4.050\end{array}$ & $0 \cdot 80$ & $1.9 \times 10^{8}$ & 34 & & & 120 & - \\
\hline
\end{tabular}

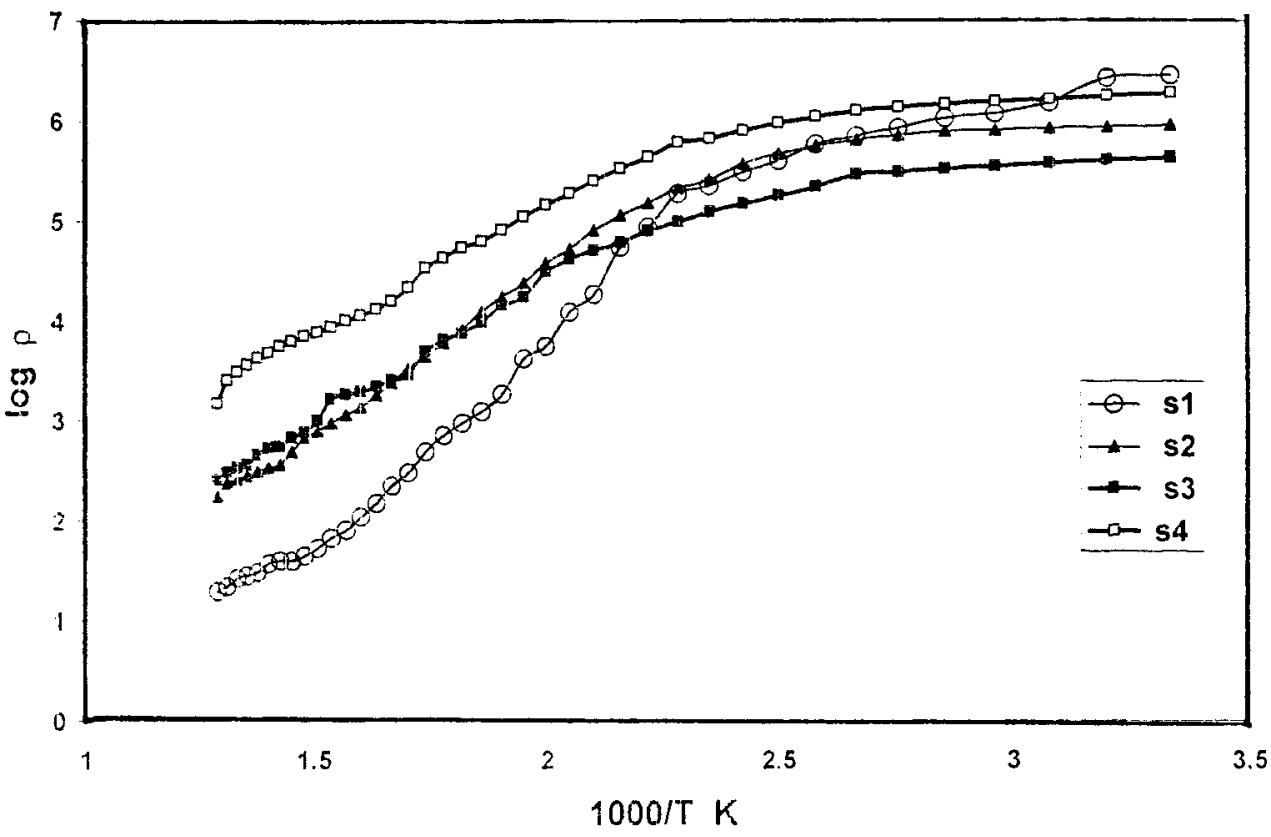

Figure 3. Variation of d.c. resistivity $(\log \rho)$ with temperature $(1000 / T) \mathrm{K}^{-1}$. 
supported by the nature of $\alpha$ vs $T$ plots. The temperature variation of conductivity in this case was mainly attributed to change of drift mobility with temperature rather than to the variation of charge carrier concentration. According to hopping model electrons are strongly localized on the cations. The localization may be attributed to electron-phonon interaction. An additional localization of electron at $\mathrm{Fe}^{2+}$ ions is over octahedral site in the spinel lattice (Austin and Mott 1969).

The frequency variation of dielectric constant $\left(\varepsilon^{\prime}\right)$ for present composites at room temperature is shown in figure 6.
From the figure it can be seen that all composites show dielectric dispersion. In ionic crystals the dielectric constant $\left(\varepsilon^{\prime}\right)$ decreases rapidly with increasing frequency and then reaches a constant value. The values of dielectric constant at $1 \mathrm{kHz}$ are noted in table 1 . The higher value of dielectric at low frequency in ionic crystal is due to voids, dislocation, and other defects (Lalitha Sirdeshmukh et al 1998). However in present composites the large values of $\varepsilon^{\prime}$ have been attributed to the fact that ferroelectric grains are surrounded by ferrite grains (Appel 1968). This in itself gives interfacial polarization.

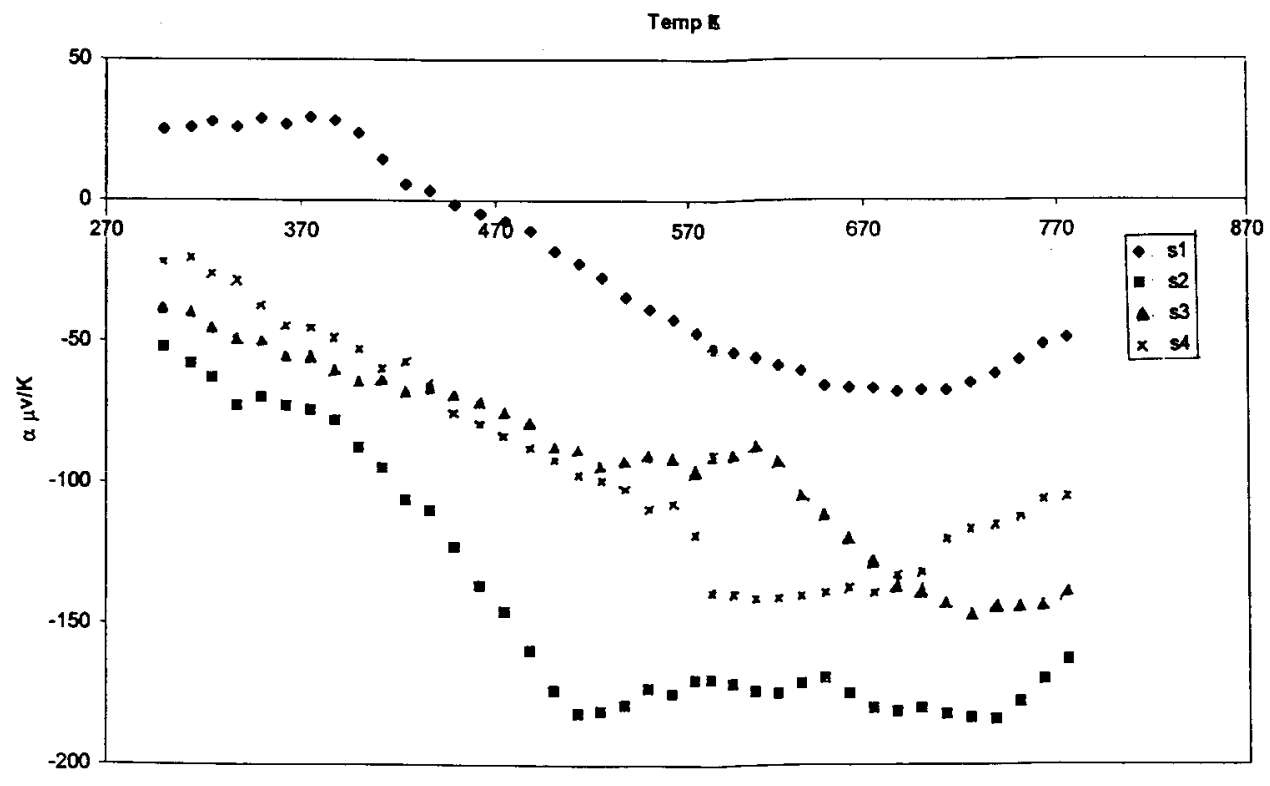

Figure 4. Variation of Seebeck coefficient $(\alpha)$ with temperature $(T) \mathrm{K}$.

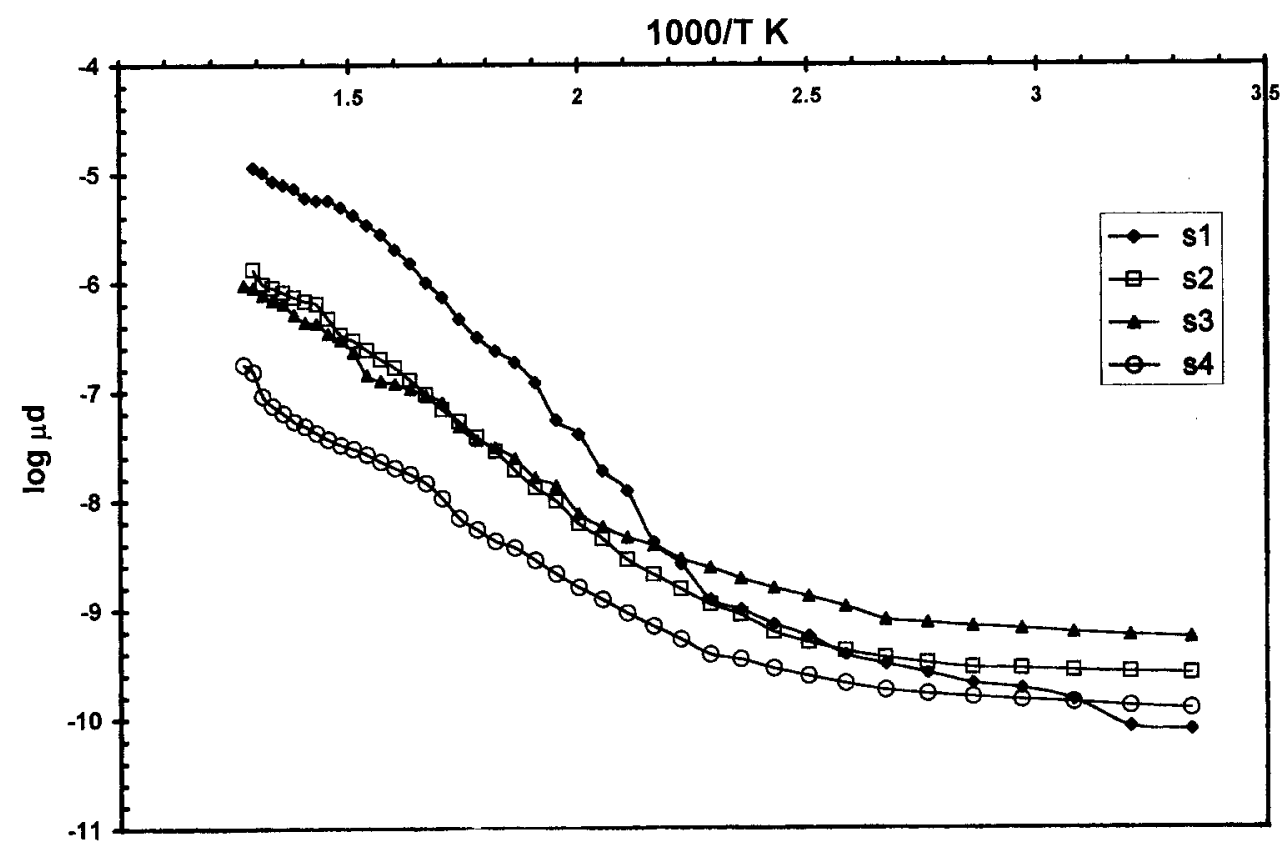

Figure 5. Variation of mobility $(\log \mu d)$ with temperature $(1 / T) \mathrm{K}^{-1}$. 


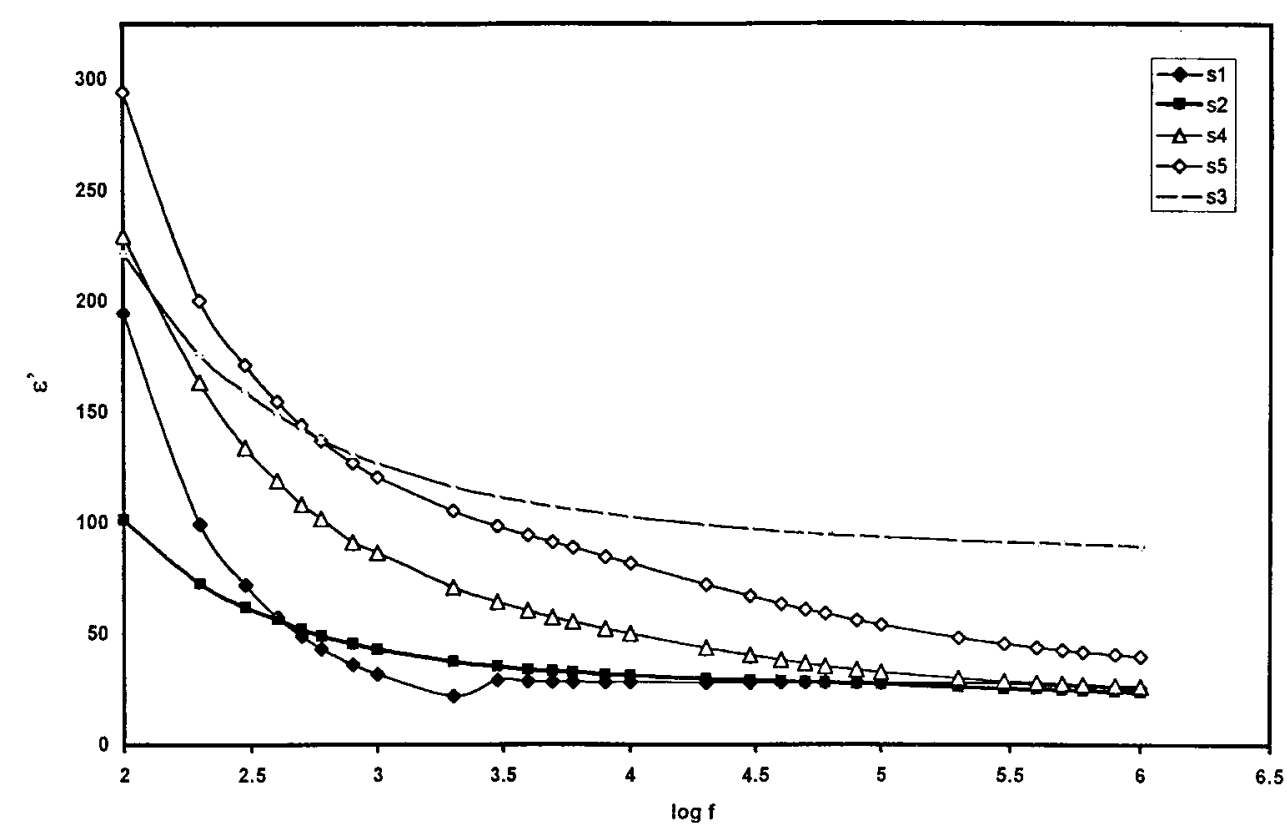

Figure 6. Variation of dielectric constant $\left(\varepsilon^{\prime}\right)$ with frequency in $\mathrm{Hz}$.

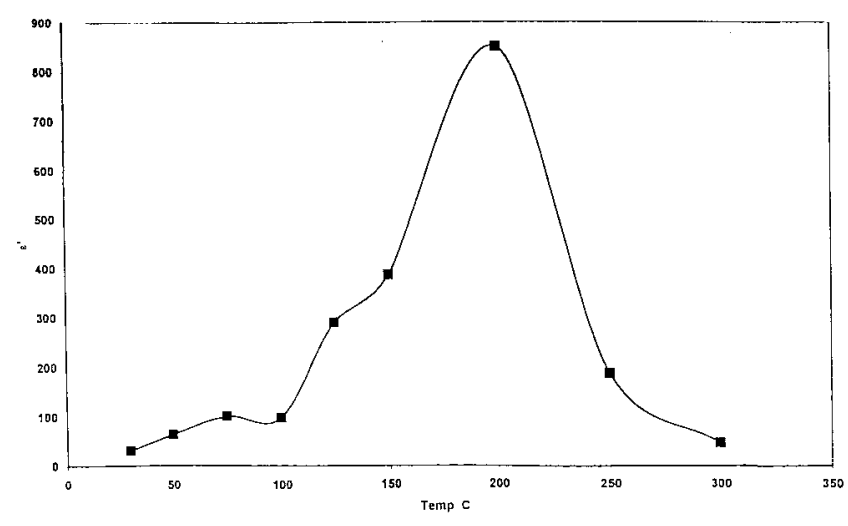

Figure 7. Variation of dielectric constant $\left(\varepsilon^{\prime}\right)$ with temperature $(T){ }^{\circ} \mathrm{C}$ for $\mathrm{CuFe}_{2} \mathrm{O}_{4}$ at $10 \mathrm{kHz}$.

The temperature dependence of $\varepsilon^{\prime}$ for $\mathrm{CuFe}_{2} \mathrm{O}_{4}$ is shown in figure 7. It is observed that the dielectric constant $\left(\varepsilon^{\prime}\right)$ shows a peak at $475 \mathrm{~K}$. This is attributed to phase transition from tetragonal to cubic in $\mathrm{CuFe}_{2} \mathrm{O}_{4}$ (Patil 1980). The temperature dependence of dielectric constant for composites is shown in figure 8, which shows two peaks for $25 \%$ mole $\mathrm{BaTiO}_{3}$ whereas for $50 \%$ and $75 \%$ of $\mathrm{BaTiO}_{3}$ only one peak is observed. The temperature of peak i.e. $400 \mathrm{~K}$, is nearly same as the Curie temperature of ferroelectric phase i.e. $\mathrm{BaTiO}_{3}(393 \mathrm{~K})$. However the peak becomes broad as the content of $\mathrm{BaTiO}_{3}$ increases. To confirm small polaron conduction we have examined the variation of conductivity with frequency at room temperature. Several workers (Appel 1968; Kumar Krishan et al 1987) have discussed conduction phenomenon in ferrites by using polaron-hopping

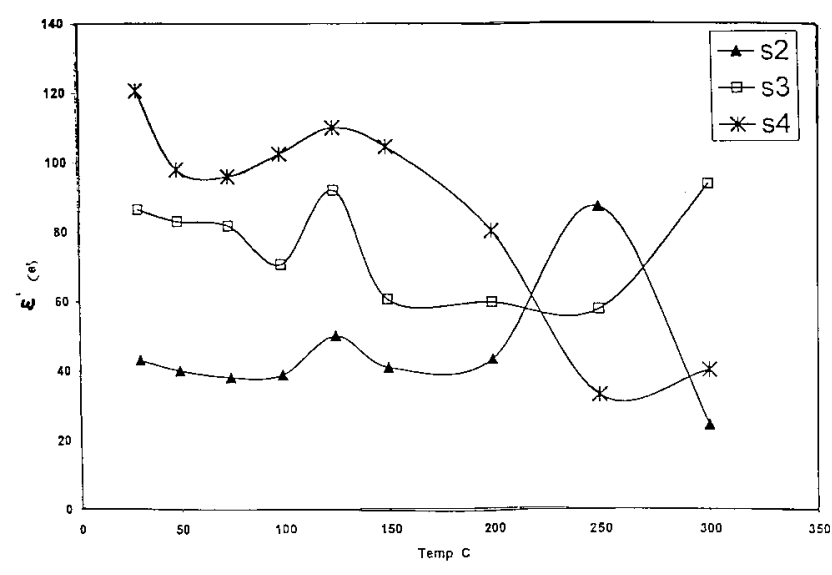

Figure 8. Variation of dielectric constant $\left(\varepsilon^{\prime}\right)$ with temperature $(T){ }^{\circ} \mathrm{C}$ for composites at $10 \mathrm{kHz}$.

model. There are two types of polarons viz. large and small polarons. In the large polaron model the conductivity decreases with frequency, at higher temperature the conduction is by thermally activated hopping mechanism.

Ac conductivity, $\sigma_{\mathrm{ac}}$ is obtained from the data on dielectric constant $\left(\varepsilon^{\prime}\right)$ and loss tan $\delta$ using the relation

$$
\sigma_{\mathrm{ac}}=\varepsilon^{\prime} \varepsilon_{0} \omega \tan \delta
$$

where $\varepsilon_{0}$ is the vacuum permittivity and $\omega$ the angular frequency.

Figure 9 shows plots of $\log \left(\sigma_{\mathrm{ac}}-\sigma_{\mathrm{dc}}\right)$ against $\log \omega^{2}$. It can be seen that plots are straight lines. This indicates that the conduction is due to small polarons. It has been shown that for ionic solids the concept of small polaron is valid (Appel 1968). 


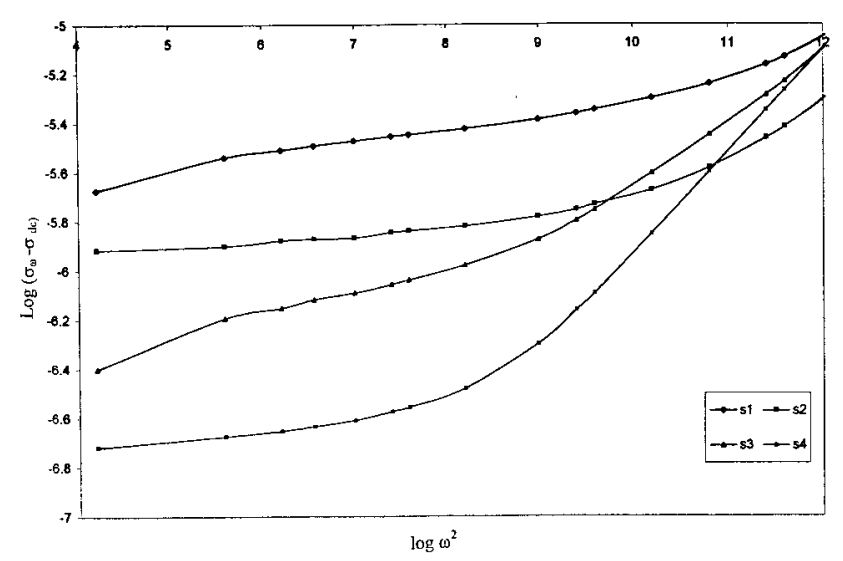

Figure 9. Variation of $\log \left(\sigma_{\omega}-\sigma_{\mathrm{dc}}\right)$ with $\log \left(\omega^{2}\right)$.

For the present composites the d.c. $\mathrm{ME}(\mathrm{d} E / \mathrm{d} H)_{\mathrm{H}}$ coefficient as a function of intensity of applied dc magnetic field was measured and maximum value obtained at $1 \mathrm{KOe}$ is noted in table 1. Magnetoelectric conversion is the result of piezomagnetic strain in spinel phase, which creates piezoelectric charge in ferroelectric phase, and hence latter would depend upon the variation of piezomagnetic coefficient with intensity of magnetic field. The polarization induced could be due to the displacement of Fe ions, thereby changing the ionic polarizability or creating quasi-permanent dipoles. The value of $(\mathrm{d} E / \mathrm{d} H)_{\mathrm{H}}$ increases with increasing barium titanate content. The low values may be due to low resistivity of ferrite phase than that of ferroelectric phase. Hence the charges which get developed in piezoelectric phase leak out through low resistance path.

\section{Acknowledgements}

Authors are thankful to DST, New Delhi for the financial support. Two of the authors (RPM and KKP) are grateful to UGC and DST for award of fellowships.

\section{References}

Appel J 1968 Solid state phy. (eds) F Seitz, D Thurnbull and Ehrenreich H 1968 (NY: Academic Press) 21 p. 193

Austin I G and Mott N F 1969 Adv. Phys. 1841

Boomgaard J, Van Den and Born R A J 1978 J. Mater. Sci. 13 1538

Ce-Wen Nan 1994 Phys. Rev. B50 6082

Eatch A I, Ghani A A, El. Shahat M F and Faramawy E I 1987 Phys. Status Solidi (a)104 793

Klinger M I 1975 J. Phys. C8 3595

Kumar Krishan C C, Wu S and Mason T C 1987 J. Solid State Chem. 37144

Lalitha Sirdeshmukh, Krishna Kumar K, Bal Laxman S, Rama Krishna A and Sathaiah G 1998 Bull. Mater. Sci. 21219

Lopatin S S 1994 Ferroelectrics 16263

Miyahara S and Kino Y 1965 Jap. J. Appl. Phys. 4310

Nanba N and Kobayashi S 1978 Jap. J. Appl. Phys. 171819

Patil S A 1980 Studies on physical properties of $\mathrm{Cu}_{x} \mathrm{Fe}_{3-x} \mathrm{O}_{4}$ ferrites, Ph.D. Thesis, Shivaji University, Kolhapur

Patil S A, Patil B L, Lotke S D and Patil R N 1996 Phase Trans. 5629

Schmid H 1994 Bull. Mater. Sci. 171411

Stierstadt K, Benz H and Rechenberg H 1964 Proc. int. conf. magnetism (Nottingham UK: Institute of Physics) p. 605

Suryanarayana S V 1994 Bull. Mater. Sci. 171259

Van Suchtelen 1972 J. Phillips Res. Rep. 2728

Verwey E J W and Heilman 1947 J. Chem. Phys. 15174 